\title{
Kernos
}

Revue internationale et pluridisciplinaire de religion grecque antique

$10 \mid 1997$

Varia

\section{Les rites funéraires dans les Suppliantes d'Euripide}

\section{François Jouan}

URL : http://journals.openedition.org/kernos/660

DOI : $10.4000 /$ kernos. 660

ISSN : 2034-7871

\section{Éditeur}

Centre international d'étude de la religion grecque antique

\section{Édition imprimée}

Date de publication : 1 janvier 1997

Pagination : 215-232

ISSN : 0776-3824

Référence électronique

François Jouan, «Les rites funéraires dans les Suppliantes d'Euripide », Kernos [En ligne], 10 | 1997, mis en ligne le 12 avril 2011, consulté le 01 mai 2019. URL : http://journals.openedition.org/kernos/660 ; DOI : 10.4000/kernos.660 


\section{Les rites funéraires}

\section{dans les Suppliantes d'Euripide*}

Les Hikétidès, qui font partie des pièces dites alphabétiques, ne sont pas regardées par la critique comme un drame du premier choix, et on a pu déplorer que celui-ci ait été conservé de préférence à certaines tragédies d'Euripide les plus célèbres dans l'antiquité, comme Andromède ou Phaéthon. On y a vu une pièce de circonstance, écrite pour relever le moral des Athéniens après une lourde défaite militaire ${ }^{1}$. L'auteur s'était proposé d'exalter à travers la personne de Thésée les vertus de respect de la justice et d'hospitalité dont se glorifiaient les Athéniens et de venger en quelque sorte sa cité en mettant en scène une victoire d'Athènes sur l'impiété des Thébains située dans un passé mythique prestigieux. Cet «éloge d'Athènes » ne serait ainsi qu'un doublet des Héraclides, composés quelques années auparavant. On lui reproche l'abus des digressions et des anachronismes et le manque d'unité dramatique : chaque scène serait traitée pour son effet propre, au détriment de la structure générale. Une fois les corps des chefs argiens rapportés par Thésée à Éleusis, l'action languit dans le dernier tiers de la pièce, tout juste animée par l'oraison funèbre que prononce Adraste et le suicide imprévu d'Evadné, imaginé simplement pour combler un vide scénique. Le vrai tragique est absent de la pièce; il est remplacé par un débordement du pathétique qui finit par lasser. Enfin, les rôles principaux, ceux d'Ethra, de Thésée et d'Adraste, manquent de relief et de substance humaine.

* Principales études consultées, outre l'indispensable édition commentée de C. COLLARD, Euripides "Supplices", 2 vol., Groningen, 1975: E. REINER, Die rituelle Totenklage der Griechen, Stuttgart-Berlin, 1938 (Tübinger Beitr., 30); G. ZUNTZ, The Political Plays of Euripides, Manchester, 1955; D.J. CONACHER, Religion and Etbical Attitudes in Euripides' Suppliants, in TAPbA, 87 (1956), p. 8-26; G. PADUANO, Interpretazione delle Supplici di Euripide, in ASNP, 35 (1966), p. 193-249; W.D. SMITH, Expressive Form in Euripides' Suppliants, in HSCP, 71 (1966), p. 151-170; R.B. GAMBLE, Euripides' Suppliant Women. Decision and Ambivalence, in Hermes, 98 (1970), p. 385-412; D.C. KURTZ - J. BOARDMAN, Greek Burial Customs, Londres, 1971; M. ALEXIOU, The Ritual Lament in Greek Tragedy, Cambridge, 1974; V. DI BENEDETTO, Euripide, teatro e società, Turin, 1978 (p. 154 193); N. LORAUX, L'invention d'Athènes, Paris, 1981; R. AÉLION, Euripide béritier d'Escbyle, 2 vol,, Paris, 1983; M.S. MIRTO, Il lutto e la Cultura delle Madri: le Supplici di Euripide, in QUCC, 47 (1984), p. 57-88; R. GARLAND, The Greek Way of Death, Cornell Univ. Press, 1985; Z. PETRE, La représentation de la mort dans la tragédie grecque, in StudClas, 23 (1985), p. 21-35; J.E.G. WHITEHORNE, The Dead as Spectacle in Euripides' Bacchae and Supplices, in Hermes, 114 (1986), p. 59-71; N. LORAUX, Les Mères en deuil, Paris, 1990; R. REHM, Marriage to Death, Princeton, 1994; E. GEORGOULAKI, Religious and Socio-Political Implications of Mortuary Evidence, in Kernos, 9 (1956), p. 95-120. - Nous citerons ces études, sauf exception, par le nom de l'auteur.

1 Voir par exemple le jugement d'A. RIVIER, Essai sur le théâtre tragique d'Euripide, Paris, $1975^{2}$, p. 155-156; G. ZUNTZ, p. 89-94; éd. COLLARD, I, p. 24-25. 
Aussi la primauté est-elle dévolue au chœur tragique des mères argiennes, et l'on retrouve la structure archaïque des Suppliantes d'Eschyle. Comme celui-ci avait déjà traité dans ses Éleusiniens le sujet des Suppliantes d'Euripide, on a même soupçonné le poète d'avoir avant tout cherché à affirmer sa supériorité sur son prédécesseur.

Ces critiques sont loin d'être toutes également fondées. Elles témoignent surtout d'une propension trop fréquente à juger d'un drame antique à l'aune de l'esthétique moderne, sans chercher à se représenter le but que visait le poète ancien. On ne peut nier, il est vrai, que l'actualité et l'idéologie tiennent une place notable dans la pièce, en particulier dans la scène entre Thésée et le héraut thébain et dans l'oraison funèbre d'Adraste : la démocratie de type péricléen y est louée, ainsi que les mérites de la classe moyenne paysanne et l'idéal civique de dévouement à l'État. Le climat reflète l'état d'esprit des Athéniens, à ce moment de la guerre du Péloponnèse, envers deux cités majeures de la Grèce: Thèbes, l'ennemie, et Argos, l'alliée potentielle, Sparte n'étant du reste qu'égrati gnée au passage (187-88).

En fait, les Suppliantes répondaient à une actualité plus immédiate encore. $\mathrm{Si}$, comme on l'admet le plus souvent, la pièce a été représentée aux Grandes Dionysies de 433, elle constitue une réplique directe au désastre subi par Athènes en novembre précédent à la frontière de la Béotie ${ }^{2}$, qui avait provoqué les pertes humaines les plus importantes depuis le début de la guerre : un millier d'hoplites avec leur stratège, sans compter les supplétifs. De plus, prenant acte du sacrilège commis par les Athéniens en se retranchant dans le sanctuaire apollinien de Délion, les Thébains avaient refusé dix-sept jours durant la trêve qui devait permettre de relever les morts. Il est difficile de ne pas voir dans les Suppliantes une protestation indignée à l'égard des Thébains, un témoignage de sympathie pour le chagrin des familles athéniennes dont beaucoup avaient des morts à déplorer, et une affirmation de la solidarité de toute la cité dans ce deuil. La légende de Thèbes offrait à cet égard un épisode trop propice pour être négligé : le refus des Thébains de rendre les corps des Argiens tombés devant les portes lors de l'assaut de la ville ${ }^{3}$. Il permettait de stigmatiser l'impiété de toujours des Cadméens et de présenter une version des faits où les Athéniens devaient recourir aux armes pour faire rendre justice aux morts. Cette intervention des Athéniens, répondant à la supplication des mères des sept chefs et du roi d'Argos, Adraste, formait l'objet du prologue et des deux premiers épisodes de la pièce. L'action y était conduite jusqu'au moment où les corps des tués, ramenés par Thésée et ses hommes du champ de bataille sur le sol attique,

2 THUC., IV, 90-99; ZUNTZ, p. 94-98; éd. COLLARD, I, p. 9-14. La principale difficulté réside dans l'intervalle très court (4 à 5 mois) entre le désastre de Délion et les Dionysies de 423. Quelques négligences matérielles ( $c f$. infra, p. 231) pourraient s'expliquer par un travail hâtif.

3 Dans la Thébaïde, les funérailles des Sept avaient lieu à Thèbes (cf. A. SEvEryns, Le Cycle épique..., Paris, 1928, p. 223-224, qui pense que la version guerrière viendrait des Épigones ou de l'Alcméonide). Adoucie dans les Éleusiniens d'Eschyle, elle était connue d'Hérodote (IX, 27). 
pouvaient recevoir les honneurs qui leur avaient été refusés. Ceux-ci allaient être détaillés tout au long des stasima et des deux épisodes précédant l'épilogue (778-1164). Ces quelque quatre cents vers constituaient à coup sûr pour Euripide et pour son public le coeur de la tragédie et son plus vif attrait, au moins sur le plan de l'émotion humaine et du pathétique. Ils répondaient aussi au goût des spectateurs, qui aimaient voir représentés les divers rites religieux qui ponctuaient leur existence : prières, sacrifices, processions de fête, rites du mariage et de la mort. Pour nous, la représentation minutieuse du déroulement des cérémonies funéraires, de la toilette des corps à l'édification du tombeau, offre une abondance de renseignements sans équivalent dans aucune autre tragédie. À ce titre, les Suppliantes ont été l'objet d'études très complètes, dont nous ferons un large usage ${ }^{4}$. Mais le but que nous nous proposons est un peu différent : nous voudrions montrer les limites de cette simulation des rites funéraires, publics et privés, de l'Athènes contemporaine, qui en offre souvent un reflet trompeur. D'abord, parce que les données du mythe héroïque thébain ne correspondent que superficiellement à la situation athénienne au lendemain du drame de Délion : les victimes sont des Argiens, tombés au cours d'une expédition normalement condamnable; leurs corps ne seront pas traités de la même façon, ni dans les mêmes lieux, les rites se partageant entre le champ de bataille thébain, Éleuthères, Éleusis et Argos. Ces obsèques relèvent à la fois du domaine des rites familiaux et privés et du domaine public, celui des cérémonies collectives qui se déroulent sous le contrôle de l'État. Enfin, comme dans nombre d'autres drames, les pratiques de la vie courante seront transposées selon les canons esthétiques de la poésie funéraire, qui ont imposé au théâtre une certaine stylisation, un modèle idéal de ce qu'on a pu appeler la «mort tragique $»^{5}$.

Car la mort est par excellence le domaine de la tragédie. Des trente-deux drames attiques du $v^{\text {e }}$ siècle que nous lisons encore dans leur intégralité ${ }^{6}$, vingt quatre incluent une ou plusieurs morts, presque toujours violentes. Dans dixneuf d'entre elles, les corps des tués sont rapportés sur la scène ou exposés aux yeux des spectateurs au moyen de l'eccyclème. Quinze pièces enfin représentent une partie au moins des rites funéraires qui aboutissent au dépôt des restes humains au tombeau, ou de ceux par lesquels on honore la mémoire des défunts : visite à la tombe, prières, libations et offrandes?

On dira, il est vrai, que cette grande ombre qui s'étend sur l'ensemble de la tragédie grecque n'est que l'expression d'un sentiment universel, l'angoisse du passage inévitable du monde des vivants dans le monde des morts. Mais cette

\footnotetext{
4 À ce point de vue, voir en particulier les études de REINER, ALEXIOU, MIRTO, GARLAND, WHITEHORNE et REHM.

5 Cf. Z. PETRE, p. 25-28.

6 En comptant le Rbésos, qui est peut-être du IV siècle.

7 Voir B. DEFORGE, La mort tragique ou tuer n'est pas jouer, in Kentron, 11-12 (1995-1996), p. 84-85.
} 
hantise prend, chez les Grecs, une coloration particulière, car elle est souvent liée à la crainte d'être privé, au moment décisif où se sépareront le corps et l'âme ${ }^{8}$, du bénéfice des rites qui permettront au défunt d'entrer dans le royaume d'Hadès. Le refus de sépulture ou le traitement outrageux du cadavre sont au centre de plusieurs tragédies ou en constituent au moins un motif important. Une conduite aussi contraire à l'éthique grecque peut se justifier par une haine exacerbée, comme celle d'Égisthe et de Clytemnestre à l'égard d'Agamemnon, ou par la cupidité et la cruauté d'un barbare comme le Thrace Polymestor, ou encore par le rejet du corps d'un guerrier considéré comme traître à son pays (Polynice) ou à ses compagnons d'armes (Ajax). Mais ce qui est exceptionnel dans les Suppliantes, c'est qu'il s'agit d'un refus de sépulture collectif, étendu à tous les morts argiens, des généraux aux simples soldats. Ce refus est mis au compte de l'enivrement de la victoire (124), c'est un acte d'bybris, témoignant d'une rancune envers les ennemis poursuivie de façon déraisonnable au-delà de la mort (525-541).

L'accomplissement des rites funèbres est bien le but et le terme de l'action des Suppliantes: c'est ce dont témoigne le retour lancinant dans la bouche des

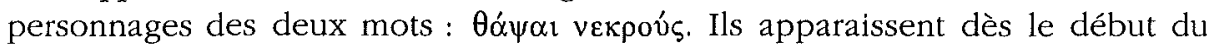
prologue (16-17) et ils figurent dix-huit fois dans la pièce ${ }^{9}$. L'action comporte trois grands mouvements : I. La supplication des mères auprès d'Athra, qui seule peut convaincre son fils Thésée d'intervenir pour obtenir la restitution des corps (1-364). II. Thésée et les Thébains : refus verbal de Créon, transmis par le Héraut et donnant lieu à un agôn; puis action militaire des Athéniens, qui s'achève par la déroute de Thèbes et l'annonce du retour des morts (754-777). III. Les funérailles des tués (778-1164). L'exodos apporte à l'action la sanction divine et relie l'épisode mythique au temps présent grâce à l'utilisation par le poète de plusieurs aitia (1165-1234).

Comme dans les Éleusiniens, l'action se situe à Éleusis, où des traditions locales plaçaient les tombes des sept chefs tombés devant Thèbes. Mais Euripide a tiré de cette localisation des effets nouveaux. D'abord, en y plaçant $\mathbb{E}$ thra ${ }^{10}$, à laquelle il est le premier à attribuer le titre d'épouse légitime d'Égée. Veuve du roi, la « reine-mère » est venue représenter les Athéniens au sanctuaire de Déméter pour célébrer la fête des proerosia ${ }^{11}$, à la veille des labours d'automne, «dans cette enceinte », dit-elle, « où pour la première fois les épis féconds jaillirent de ce sol» (30-31). C'était une manière de rappeler l'invention de

\footnotetext{
8 Voir Suppl., 532-536 et le comment. ad loc. de COLLARD. Sous cette forme, il semble qu'il s'agisse simplement de l'expression de la croyance populaire.

9 16-17, 121, 130, 174, 385, 538-539, 571, 670-671, 760, 935; cf. REHM, p. 110-111.

10 Athra n'est jamais présente à Athènes. Dans les Chants Cypriens, elle vit à Aphidna lorsque Thésée enlève Hélène enfant (fr. 13 Bern.).

11 Sur cette fête, voir J. CASABONA, Recherches sur le vocabulaire des sacrifices en Grèce, Paris, 1966, p. 104-108; A.Ch. BRUmfield, The Attic Festivals of Demeter..., New York, 1981, p. 54 sq.
} 
Triptolème, dont les Athéniens étaient si fiers. De plus, le public savait que cette fête précédait de peu les Théseia ${ }^{12}$, eux-mêmes suivis des Epitaphia, où l'on commémorait les Athéniens morts au combat dans l'année et où l'on prononçait leur oraison funèbre ${ }^{13}$. Mais le poète ne vise pas seulement à intégrer l'action dans le calendrier religieux de la cité. La localisation au sanctuaire d'Éleusis reste très marquée dans la première partie de la pièce et il y sera fait de nouveau référence lors de l'épisode d'Evadné et dans l'exodos. L'autel des deux déesses où Æthra est venu sacrifier est cité à plusieurs reprises $(1 ; 32-33 ; 64-65 ; 93)$. Il se trouve dans l'enceinte sacrée ( $\sigma \eta \kappa \kappa^{\prime} \varsigma$ ) au fond de laquelle se dresse la façade du

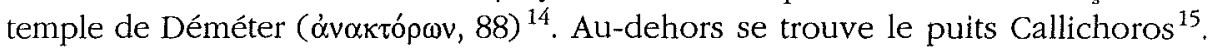
Ce lieu est le foyer de Déméter-Deiô $(1 ; 290)$, de la déesse porte-torche $(260)$, le saint parvis de Perséphone (217). C'est le site de la célébration des mystères (173). À la fin de la pièce sera localisé dans cette enceinte le bûcher de Capanée, au pied de la falaise d'où se jettera Evadné, et à proximité se trouve le lieu de crémation des autres chefs ${ }^{16}$.

Dès le début du drame, contrairement aux usages tragiques, scène et orchestra sont remplis de personnages immobiles : le Chœur des Mères accompagnées de leurs servantes, le Chœur secondaire des fils des Sept; Adraste, les prêtres du sanctuaire ${ }^{17}$. Tous entourent $Æ$ thra, debout devant l'autel. On pourrait croire que l'on assiste au déroulement régulier de la liturgie en l'honneur de Déméter, ce que contredisent les tenues de deuil et les pleurs des Mères, qui font tache dans le climat de fête qui devrait régner ${ }^{18}$, ainsi que l'attitude accablée des autres assistants (Adraste est enveloppé dans son manteau, 110). La réalité est qu'il s'agit d'un sacrifice interrompu. Qui plus est, ce n'est pas l'aide des déesses que le Chœur est venu implorer, mais celle de la mère de Thésée, qui préside à la cérémonie.

Malgré le caractère imposant et émouvant de ce tableau, il n'est pas moins vrai que les Mères ont commis une grave faute religieuse, en interrompant le déroulement du rite éleusinien, qui reste en fait inachevé. Nous savons par

12 C. CALAmE, Thésée et l'imaginaire atbénien, Lausanne, 1990, p. 153-156.

13 N. LORAuX, L'invention..., passim.

14 Il s'agit en fait du Télestérion : les deux autels jumeaux étaient placés sur la terrasse située devant l'édifice. Cf. J. CARrière, Le Cbceur secondaire..., Paris, 1977, p. 51 sq.

15392,619 . Placé à l'entrée du sanctuaire, il sert de point de ralliement aux troupes de Thésée.

$16980-989$.

17 R. REHM, The Staging of Suppliant Plays, in GRBS, 29 (1988), p. 263-307, chiffre (p. 275) le nombre des personnes présentes entre 23 et 32 , et en tire une conclusion fortement argumentée en faveur de l'identité des autels des déesses avec l'autel central de l'orchestra. Les mouvements des personnages et des choreutes, qu'il analyse jusqu'à la fin du second épisode, comporteraient des échanges entre orcbestra et skénè beaucoup plus nombreux que dans l'usage tragique habituel. Figuration importante aussi pour le second retour des corps (WHITEHORNE, p. 68).

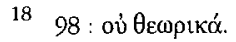


Andocide ${ }^{19}$ que le fait de perturber les rites d'Éleusis était sévèrement puni. Les Mères, et Adraste après elles, reconnaissent du reste qu'elles ne sont pas venues là pour s'associer "pieusement ${ }^{20}$ au culte mais pour y joindre Æthra et l'implorer. Il ne s'agit donc pas de célébration, mais de supplication ${ }^{21}$. Mais la douleur des mères, qui s'exprime de façon poignante dans la parodos, constitue une excuse valable pour cette entorse à la liturgie. Même, du fait qu'elles se jettent « par nécessité, en suppliantes, au pied des autels divins accueillants à la flamme » (63-64), elles renforcent la justice de leur cause (65) par une caution sacrée, celle de la puissante déesse. Car si Æthra est implorée comme la mère de Thésée, Déméter est aussi une mère, qui a longtemps et douloureusement souffert de la disparition de sa fille, entraînée dans le monde souterrain. Ce sera plus tard le sort d'Evadné, qui s'élancera «vers les chambres de Perséphone ${ }^{22}$. Dès lors, tout le début de la pièce se place sous le signe des Mères, et l'on n'entendra jusqu'à la fin de la parodos que des voix féminines.

Les motifs funéraires sont concentrés dans la parodos et les quelques vers qui suivent (42-97), une parodos qui n'est en fait que le premier chant du chour, puisqu'il est sur place depuis le début. La plainte des mères n'est pas un véritable thrène rituel; il n'en est qu'une ébauche, puisque les corps ne sont pas présents, comme dans la protbésis des funérailles privées ${ }^{23}$. Les Mères sont ici dans la situation des femmes d'Athènes, lorsqu'elles eurent appris que leurs maris ou leurs fils étaient tombés devant Délion. Aussi les Argiennes se lamentent-elles de savoir les corps de leurs enfants abandonnés, en proie aux bêtes sauvages, privés de tombe ${ }^{24}$. Les gestes traditionnels - exposer le corps à la maison, verser la terre d'un tombeau - sont évoqués sous une forme négative (52-53). Mais les paroles de Thésée à son entrée en scène attestent que ces femmes sont déjà en position de pleureuses rituelles. Le crâne rasé, couvertes de vêtements noirs (97), elles se livrent à toutes les manifestations extérieures du deuil (94-95) : elles se déchirent les joues de leurs ongles, se frappent la poitrine, versent des flots de larmes, et surtout leurs plaintes ne laissent aucun doute sur la nature de leurs chagrins: elles exécutent le «thrène pour les morts»(88). C'est le rite par excellence dévolu aux femmes lors des funérailles, pour lequel Euripide emploie plus

19 I, 113 et 116; voir les notes de l'éd. DaLmEYDA, p. 138, 1 et 4.

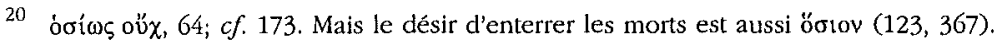

21 Sur ce motif chez Euripide, voir AÉLION, II, p. 18-31. Il se combine avec un autre motif très prisé d'Euripide, celui du « recours à l'autel».

22 1022. Sur l'atmosphère de sacré éleusinien de la pièce, voir PADUANO, p. 227-228; REHM, p. 110-111. Il ne semble pas, en revanche, malgré B. LAVAGNINI (AJPb, 68 [1947], p. 83-84), que les vers 54 et 469 renferment des allusions aux rites d'Éleusis.

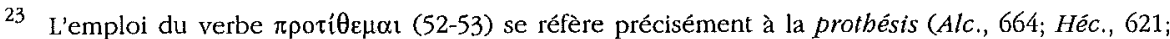
Pbén., 1319).

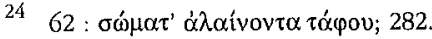


souvent le terme de fóos ou $\gamma^{\prime}$ o $^{25}$. Ce chant funèbre du chœur tragique s'accompagne d'une danse au rythme approprié, "le chœur que célèbre Hadès » ${ }^{26}$. Par ce chant entremêlé de sanglots et rythmé par les gestes des participants, la douleur s'extériorise et en même temps s'atténue, comme bercée par le ton incantatoire et les échos entre strophe et antistrophe. Cette sorte de soulagement est exprimé par le Chœur, lorsqu'il évoque la «volupté des sanglots », un sentiment qui, selon le mot d'un critique, parcourt toutes les parties lyriques des Suppliantes ${ }^{27}$.

Dans cette première phase, on voit déjà apparaître quelques thèmes traditionnels de la déploration funèbre qui seront développés par la suite : la révolte devant l'inversion des lois naturelles, qui fait qu'une mère est obligée d'ensevelir son fils (174-75); l'angoisse devant la vieillesse stérile qui attend la mère frustrée de son enfant (169-170); son désir lancinant de serrer une dernière fois dans ses bras le corps du fils qu'elle a mis au monde (69-70); enfin le vœu - qui ne cessera d'être répété par la suite - de mourir pour « oublier une souffrance intolérable » (86). On remarquera en passant deux termes rares employés pour définir les rites funèbres : $\dot{\omega} \rho \alpha \hat{\imath} \alpha$ (175) : proprement «ce qui vient à son heure », et $\kappa \tau \varepsilon \rho i ́ \sigma \mu \alpha \tau \alpha$ $(309)^{28}$, qui désigne au sens restreint les offrandes déposées dans la tombe, mais qui s'étend ici à tout le rituel funéraire.

Il ne faut pas moins du tiers de la pièce pour que Thésée accepte de contraindre les Thébains à observer cette «loi non-écrite » concernant les morts au combat. Les supplications pressantes des Mères avaient cependant ému Æthra ${ }^{29}$. Mais la décision dépendait de Thésée. Au cours du premier épisode, le roi soumettait Adraste à un questionnaire sans complaisance. Convaincu de s'être lancé dans une expédition injuste à la suite d'un enchaînement d'erreurs et de fautes, le roi d'Argos se voyait refuser l'aide de Thésée. C'était seulement dans l'épisode suivant que la reine obtenait le revirement de son fils, en se fondant moins sur des contraintes rituelles que sur des considérations de piété, de justice et de haute exigence morale. Il y allait aussi, ajoutait-elle, de la gloire de Thésée et de la réputation de sa ville. La décision prise, les bandelettes qui «liaient» symboliquement Æthra étaient retirées, et la mère et le fils sortaient de la scène (359-360).

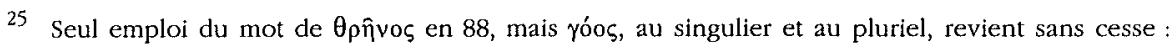

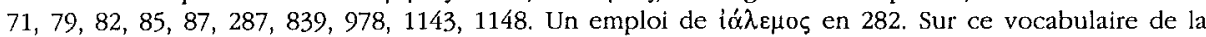
déploration, voir AlEXIOU, p. 102-103.

26 75. Cf. Hér, 1027; IT, 182. C’est le "péan des morts" (Alc., 424; Hél., 178).

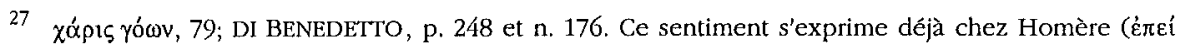

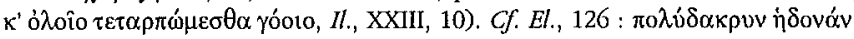

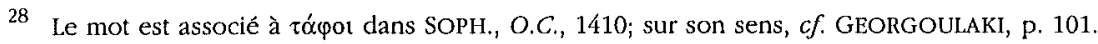

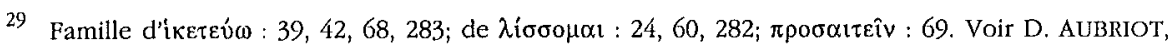
Prière et conception religieuse en Grèce ancienne, Lyon, 1992, p. 485-488. 
Dans les deux épisodes suivants, le motif de l'enterrement des morts semble passer quelque peu au second plan : le centre du premier (381-597) est un débat d'ordre politique entre le héraut thébain et Thésée; le centre du second (634-777) est un récit circonstancié de la victoire des Athéniens sur les Thébains. Mais chacun d'eux à sa manière fait progresser l'action vers le but attendu, le retour des tués. Dans un premier temps, il s'agit surtout de la justification théorique de la décision de Thésée. Accorder la sépulture aux morts est une obligation imposée par les dieux : Athra l'avait affirmé (19; 311), Thésée le confirme (563). Donc, en favoriser l'application est un devoir de piété, ce qui est proclamé à plusieurs reprises ${ }^{30}$. De plus, c'est encore une obligation reconnue par tous les hommes, c'est « la loi de tous les Grecs »" La loi morale est aussi politiquement utile, parce qu'elle contribue à la stabilité des cités ${ }^{32}$. Donc, les morts ont droit à un sépulcre $(309 ; 531-36 ; 539 ; 671-72)$, et le leur refuser, c'est outrager les hommes et les dieux, et selon la forte expression du Chœur, «souiller les lois humaines $»^{33}$. Thésée, devant Adraste, avait durement condamné l'expédition des Sept et n'avait pas contredit sur ce point le héraut thébain ${ }^{34}$. Mais la mort a en quelque sorte purifié les Argiens de leur folie. La haine des Thébains ne se justifie plus : maltraiter les morts pour punir les vivants est une conduite condamnable ${ }^{35}$. La loi commune des Grecs exige que les corps soient, conformément aux rites, ensevelis dans cette terre d'où ils viennent et où ils doivent retourner ${ }^{36}$. Mais elle ne précise par le lieu où ils doivent être inhumés. Cela peut être sur le champ de bataille même ${ }^{37}$. C'est ce que suggère Thésée lorsqu'il imagine que les Thébains pourraient s'effrayer de voir les ennemis ensevelis dans leur territoire. Il insinue même que les gens de Thèbes pouvaient redouter que ces morts ne se multiplient sous la terre (543-48) : une superstition bien digne du cerveau obtus des Béotiens, et qui pouvait faire ricaner le public d'Athènes! On peut craindre, toutefois que Thésée, cet esprit libre, ait été un peu loin, car la crainte des morts n'était pas inconnue chez les Athéniens, surtout lorsqu'il s'agissait de morts puissants comme les héros argiens ${ }^{38} \ldots$

Le Chøeur, suspendu à la décision de Thésée, puis au succès de son combat, avait émis le vou qu'il puisse « recouvrer le sanglant sujet d'orgueil d'une

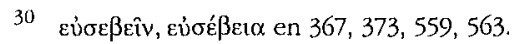

$31526,671$.

32 539-541 : supprimer cette «loi », c'est décourager les braves ( $\alpha$ $\lambda \kappa \iota \mu o 1)$, les pousser à la lâcheté, alors que le but de la cité est d'« enseigner le courage » (909-917); cf. R. GoOssENS, Euripide et Atbènes, Bruxelles, 1962, p. 449.

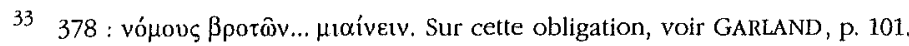

34 494-505 et 522,530 .

35 537-538; cf. SOPH., Aj., 1344-1345.

$36 \quad 531-536$.

37 C'est l'usage homérique : $c f . I l .$, VII, 430-432; GARLAND, p. 92-93.

38 Cf. Crespbonte, fr. $450 \mathrm{~N}^{2}$; GARLAND, p. 103.
} 
mère », expression qu'il reprenait à la fin du stasimon suivant : « Rapporte... l'orgueil et le soutien de la cité [de Zeus], jusqu'au bûcher, le corps outragé ${ }^{39}$ ». La victoire athénienne confirme le bien-fondé de l'initiative de Thésée et tranche définitivement le débat qui avait partagé le Chœur sur l'efficacité de la justice divine ${ }^{40}$. Adraste, rabroué par Thésée (513), s'était tu pendant plus de deux cents vers. Il prend la parole pour une complète amende honorable, puis interroge le Messager. Très vite, la stichomythie porte sur «l'enjeu du combat », c'est-à-dire les corps des victimes (754). Adraste apprend que les simples soldats ont été ensevelis par Thésée dans «les creux du Cithéron » (757). Il fait préciser - ce qui n'est pas indifférent pour les Athéniens - que c'est sur le versant attique, à Éleuthères, donc en sol ami. Euripide se contente de suivre une tradition locale, déjà adoptée par Eschyle ${ }^{41}$.

Plus surprenant est le propos du Messager sur l'attitude de Thésée à l'égard des corps des chefs. Auparavant, le héraut lui avait rappelé qu'il n'avait « aucun lien avec $\operatorname{Argos}^{42}$ ", et que le sage devait borner ses soins à ses enfants, à ses parents et à sa patrie ${ }^{43}$. Pourtant, le héros n'a pas hésité à donner à ces corps décomposés par un long abandon les soins qui seraient revenus à des esclaves (763), ou au mieux aux femmes de la famille : il les a lui-même relevés, il a lavé leurs blessures; les a couchés sur des civières et recouverts d'un tissu ${ }^{44}$. Et comme Adraste s'étonne que Thésée se soit soumis à cette besogne «affreuse et

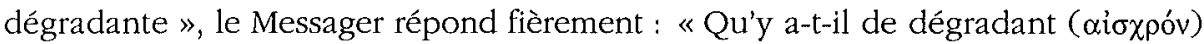
chez les hommes à partager les maux de ses semblables ${ }^{45}$ ? ». Cette attitude n'a pas surpris seulement Adraste, mais beaucoup de critiques modernes et suscité des explications parfois singulières. Thésée aurait voulu marquer qu'il négligeait la souillure de la mort, mais cet aspect de $\mu$ ía $\sigma \mu \alpha$ est étranger au texte, qui ne

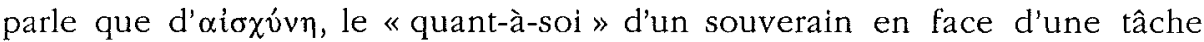
indigne de lui. On a prétendu qu'en assumant un rôle normalement dévolu aux femmes, Thésée s'était en quelque sorte «féminisé ${ }^{46}$ », mais la suite de la pièce ne justifie nullement cette thèse. On a encore mis son geste au compte de la

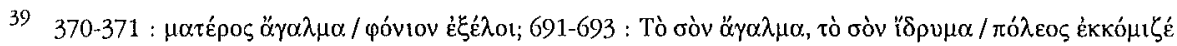

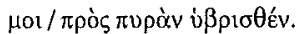

$40 \quad 608-617 ;$ cf. 731-733.

41 Salaminiens, fr. $18 \mathrm{~N}^{2}$ (Philochore, $328 \mathrm{~F} 112$ Jacoby). Les simples soldats sont donc inhumés tandis que leurs chefs sont incinérés. Les deux usages coexistent à Athènes au $v^{e} s$. On peut dire que vu le nombre des premiers, l'inhumation est plus expéditive. On a tout de même l'impression que l'incinération suivie de l'ensevelissement individuel des cendres sous un tombeau est un traitement plus «noble».

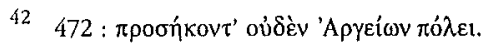

$43506-507$.

44 Pour ce traitement des corps, on comparera El., 1227; Tr., 1152; Phën., 1431 (bain et lavage des blessures); $A l c ., 613 ; \operatorname{Tr} ., 1143$ (suaire).

45 767-768; voir le commentaire de GAMBLE, p. 391-393.

46 REHM, p. 27. 
tendance de la démocratie athénienne à s'arroger les rites funéraires qui étaient jusque-là l'apanage des familles et dévolus à la parenté féminine ${ }^{47}$. C'est chercher des raisons bien loin. En l'absence des proches des défunts, un tiers généreux et amical était en droit d'accomplir les gestes les plus urgents du rituel funéraire. On peut estimer, il est vrai, que Thésée dépasse le cadre de ses strictes obligations, mais c'est une façon de montrer chez lui de nouvelles vertus qui s'ajoutent à celles dont il a déjà fait preuve : piété, vaillance, modération dans la victoire. Une telle marque d'humanité, dont la tragédie attique n'offre guère d'équivalent, a conduit certains critiques à parler de « préfiguration du franciscanisme ${ }^{48}$ ». Il y a aussi dans son geste un souci de ménager la sensibilité des vieilles mères qui se manifestera encore plus tard (944 et 946). L'attitude de Thésée s'inscrit du reste dans la tradition de l'Iliade. Achille, sans y participer, il est vrai, dirige au moins les gestes de ses compagnons autour du corps de Patrocle : «Ils lavent le corps, le frottent d'huile luisante..., le déposent sur un lit et, de la tête aux pieds, ils le recouvrent d'un souple tissu ${ }^{49}$ ». Au chant XXIV, Achille encore, après avoir fait apprêter de même le corps d'Hector par les servantes afin de ménager la douleur de Priam, prend ce corps dans ses bras pour le déposer sur le char du vieillard (582-89).

Lorsque le cortège entre, portant les cadavres des chefs sur des civières, Adraste les salue, le bras tendu, la paume en avant, un geste dévolu aux hommes, que l'on voit représenté sur les scènes de protbésis ${ }^{50}$.

À partir de ce moment, les parties lyriques, jusque-là peu importantes, vont se multiplier et s'étendre, équilibrant en volume les parties dialoguées, et les surpassant même largement si on y intègre les prologues anapestiques qui ne constituent parfois qu'un pont entre deux cantica. Ces chants sont de forme variée : deux stasima (778-793 et 955-975); deux commoi, l'un entre Adraste et le chœur principal (798-836), avant le quatrième épisode, l'autre entre le chœur des mères et le chour secondaire des enfants (1114-1164), faisant fonction de stasimon avant l'exodos; la monodie d'Evadné (990-1030); enfin deux morceaux plus brefs : un couplet lyrique du chœur (918-924) et quelques vers de commos d'Iphis avec le chour (1072-1079).

Rappelons brièvement la suite des rites funéraires qui remplissent cette partie du drame. Après le stasimon dans lequel le Chœur salue l'arrivée des corps, les civières sont posées sur le sol et Adraste et les Mères échangent les plaintes qui sont de règle lors de la prothésis attique. À peine se sont tus les

47 Voir N. LORAUX, L'invention..., p. 48-49; 66; WHITEHORNE, p. 69-70; S. GOLDHILL, The Great Dionysia and Civic Ideology, in JHS, 107 (1987), p. 67-68. Thèse contestée par GAMBLE, p. 391; REHM, p. 116.

48 G. MURRAY, cité par PaDUANO, p. 218. Thème proche dans And., 421-422, mais les autres références proposées ne sont pas valables, car il s'agit toujours de rapports entre $\varphi$ í̀ot.

49 II., XVIII, 346-353.

50 772; voir ESCH., Cho., 8-9; Alc., 767; KURTZ-BOARDMAN, PI. 35. 
derniers sanglots que Thésée, qui agira désormais comme une sorte de maître des cérémonies, invite Adraste à prononcer l'oraison funèbre des cinq chefs dont les corps sont présents : Capanée, Étéoclos, Hippomédon, Parthénopée et Tydée (857-917). Suit une nouvelle et brève explosion de douleur des Mères (919924). Thésée complète alors l'éloge funèbre en évoquant les deux autres chefs dont les corps, le public le savait, n'avaient pu être ramenés de Béotie, Amphiaraos et Polynice.

Le roi d'Athènes indique ensuite quel sera le reste de la cérémonie: des bûchers seront édifiés, l'un pour Capanée, l'autre pour le reste des chefs. Avant le départ des civières, les mères, qui auraient voulu embrasser leurs enfants une dernière fois, en sont empêchées par Thésée, pour la raison qu'« elles mourraient à les voir ainsi défigurés ${ }^{51} \gg$. Elles doivent donc se résigner et attendre que les restes des êtres chers leur soient rendus, sous forme de cendres dans les urnes funéraires. Cette frustration supplémentaire accroît l'intensité du chagrin qu'elles expriment dans le stasimon suivant (955-919).

Ce temps où la scène resterait vide est comblé par l'épisode du suicide d'Evadné, la veuve de Capanée. Après une monodie passionnée, elle se jette dans le bûcher où se consume le corps de son mari. Son vieux père, Iphis, qui n'a pu empêcher son geste dramatique et qui vient de perdre à la fois son fils Étéoclos, son gendre et sa fille, quitte la scène désespéré pour attendre la mort (980-1113).

Les anapestes du Chour annoncent le retour du cortège qui rapporte aux vieilles mères, chancelantes et appuyées sur leurs servantes, les urnes que portent les enfants des tués. Ceux-ci étaient présents depuis le début de la pièce (106), groupés autour d'Adraste, mais ils étaient restés jusque-là silencieux. Ils entonnent, en répons avec le chœur principal, un dernier thrène (1165-1234), où l'expression du chagrin et du désespoir est poussée au paroxysme.

Dans cette longue séquence rituelle, quelle était la part des éléments familiers aux Athéniens, ceux dans lesquels ils retrouvaient le reflet de pratiques quotidiennes, qu'il s'agisse des actes ou des thèmes de lamentation, et quelle était la part de transposition dans le monde du mythique, de l'imaginaire ou de la tradition poétique ? C'est à cette question que nous essaierons maintenant de répondre, dans la mesure du possible.

Euripide déploie dans les Suppliantes le goût pour les grands mouvements processionnels à travers l'orchestra qu'on retrouvera dans les deux Iphigénies ou dans Hélène. On ne relève pas ici moins de trois cortèges : celui qui rapporte sur des brancards les corps des tués; celui qui les conduit vers le lieu de la

51946 : ị $\lambda \lambda$ otwuévouc. En 941-947, Adraste avait accédé à la demande des mères de s'approcher des civières pour embrasser leurs enfants une dernière fois, mais il cède aussitôt à l'objection de Thésée. Le poète paraît vouloir opposer la raison de Thésée à la légèreté d'Adraste. Mais on a noté qu'à la fin des Baccbantes, Agavé recomposait elle-même le cadavre démembré de son fils ( $C b r$. Pat, 1466 sq.). 
crémation, accompagnés de Thésée, d'Adraste et des enfants; celui enfin qui ramène dans la même formation les enfants porteurs des urnes cinéraires.

Les deux premiers cortèges semblent plutôt appartenir au domaine des rites privés. On estime en effet qu'au $\mathrm{v}^{\mathrm{e}}$ siècle, les morts tombés sur le territoire attique ou près des frontières (ce qui était le cas des morts de Délion) étaient rapportés à Athènes pour être remis à la famille en vue des funérailles ${ }^{52}$, dont le premier acte était la prothésis. Sur ce point, la mise en scène devait serrer d'assez près la réalité. Le second cortège évoque, lui, le rite de l'ecpbora, le transport des corps vers le cimetière, où avait lieu soit l'inhumation, soit la crémation. Le cortège était essentiellement masculin, mais la présence des femmes - au moins âgées et proches parentes - n'était pas exclue ${ }^{53}$.

Dans les Suppliantes, nous l'avons vu, Thésée n'avait pas permis aux Mères de se joindre au cortège : est-ce un simple expédient théâtral pour éviter que la scène ne reste vide, ou l'usage pouvait-il justifier ce refus ? Il est difficile d'en décider. En tous cas, la coutume attique autorisait une ultime déploration à la tombe : elle est remplacée ici par le second commos.

En revanche, le cortège qui rentre en scène avec les ossements évoque plutôt le cadre les rites publics, qui prévoyaient que les morts tombés en terre étrangère étaient incinérés sur place et que leurs restes étaient rapportés à Athènes ${ }^{54}$. Ce retour, comme celui des corps, pouvait être accueilli par un thrène comportant des éléments d'éloge individuel. Du fait des nécessités de l'action, les deux moments sont fondus en un seul : la cérémonie privée, improvisée au retour des restes des morts, et l'oraison funèbre publique prononcée une fois par an à date fixe devant tout le peuple. Les cendres étaient alors placées dans un tombeau collectif sur place. Celles des chefs argiens seront naturellement inhumées par leurs proches dans leur patrie.

L'arrivée des corps déchaîne chez les mères un tumulte d'émotions opposées : soulagement «inespéré » de revoir le corps de leurs fils - un «beau spectacle» - et en même temps « la pire de toutes les douleurs » (782-785).

C'est le point de départ d'un cérémonial que nous analyserons dans l'ensemble de l'épisode, en commençant par les actes et les comportements. À ce point de vue, les textes reprennent et complètent les motifs de la parodos : le vêtement de deuil des femmes, les ongles qui déchirent les joues, la cendre que l'on répand sur la tête, les larmes, les sanglots, les gémissements mille fois répétés. C'est la participation traditionnelle des femmes aux funérailles. Mais Adraste lui-même «verse des flots de larmes » (772-774; 800-801; 838-839). On pense à Achille sanglotant auprès du corps de Patrocle ${ }^{55}$. Les mères se trans-

52 GARLAND, p. 92. Mais nous manquons de texte d'appui.

53 Sur l'ecpbora, KURTZ-BOARDMAN, p. 144-146; GARLAND, p. 31-34.

54 Voir GOLDHILL, att. cit. (n. 47), p. 67

55 Il., XVIII, 231-236; 316-317. 
portent aussi par la pensée dans leur foyer d'Argos, où elles célébreront les "rites douloureux du souvenir », répétition des gestes sacrés du jour des obsèques : boucles de cheveux déposées sur le corps, couronnes placées sur sa tête, libations à la tombe (973-975), incantations funèbres " que rejette Apollon ${ }^{56}$, et toujours des pleurs, des sanglots que, dès l'aurore, à l'imitation de Procris, elles feront entendre, en mouillant de larmes leur vêtement (977-979).

Un autre rite de la prothésis n'aura pas pu être accompli, ce contact direct des femmes de la famille avec le corps du mort que nous montrent en particulier les peintures de vases, avec ces mains qui se posent sur la tête ou sur le corps du défunt ${ }^{57}$. Ce contact physique et charnel auquel elles aspirent (815-818) leur a été refusé, et elles ne trouveront qu'une faible compensation à serrer contre leurs seins l'urne contenant les cendres de leur fils ${ }^{58}$. Il faut que le père d'Étéoclos soit plongé dans un désespoir total pour que la perspective de toucher les ossements de son fils ne lui apporte aucun soulagement (1107).

Deux épisodes coupent le flot des déplorations lyriques : l'oraison funèbre et le suicide d'Evadné. Le discours d'Adraste est peut-être la partie du drame qui a suscité le plus de commentaires et d'appréciations divergentes, sur le fond comme sur la forme ${ }^{59}$. Nous ne retiendrons ici que ce qui intéresse notre sujet. À partir du moment où Euripide reproduisait le cérémonial funèbre athénien pour les soldats morts au combat, il se devait d'y introduire l'éloge funèbre prononcé au terme de chaque année de guerre au moins depuis $464^{60}$. Formellement, le schéma en est ici assez respecté pour sembler familier aux Athéniens, même transposé à des guerriers argiens des temps mythiques. Car il n'y a guère que les noms de ces héros pour rappeler l'épopée thébaine. Le propos semble s'adresser au moins autant aux jeunes gens présents au théâtre de Dionysos qu'aux jeunes guerriers qui entourent Thésée. Le lieu même paraît insensiblement s'être transporté de l'Éleusinion au cimetière du Céramique à Athènes. Il existe, il est vrai, des différences sensibles entre notre discours et les canons oratoires de cette éloquence officielle. Celles-ci sont été bien analysées et il nous suffira de les résumer ${ }^{61}$ : Adraste omet les références aux actes de bravoure des morts, sur l'injonction formelle de Thésée (846-856), et les consolations d'usage aux familles et aux survivants; en revanche, il procède à des éloges personnels, qui

56 976-977. Il y a combinaison des rites des obsèques (dont certains, comme l'offrande de cheveux, n'ont pu être accomplis) et des rites du souvenir et de la visite au tombeau ( $\chi 0 \alpha i ́$ ).

57 Pour l'iconographie, voir KURTZ-BOARDMAN, Pl. 37; GARLAND, fig. 7.

58 Pour un bon commentaire de cet élément du rituel, voir MIRTO, p. 57-65. Au v. 1160, $\alpha \mu \varphi \mathrm{i}$

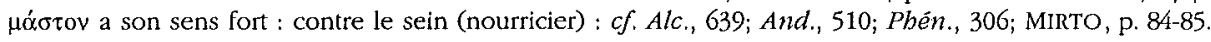

59 Sur les problèmes de ce discours, voir G. ZUNTZ, p. 13-16; PADUANO, p. 122-126; C. COLLARD, in BICS, 19 (1972), p. 39-46; N. LORAUX, L'invention...; I. MORRIS, Death Ritual and Social Structure in Classical Atbens, Cambridge, 1992, p. 131 sq.; ROEHM, p. 111.

60 La date n'est pas certaine. Voir C.W. ClaIRMONT, Patrios nomos, in Athens during the vith and the IVtb century, Londres, 1983.

61 Cf. C. COLLARD, op. cit., p. 43-45. 
sont du domaine de l'eulogie à la tombe, très ancienne pratique des funérailles privées $^{62}$. Adraste respecte cependant les normes morales et civiques de l'oraison funèbre athénienne en célébrant chez les cinq représentants de la Grèce des héros, non des vertus guerrières, mais sous des formes diverses les mérites de citoyens exemplaires, auxquels une éducation réussie a appris à se dévouer aux intérêts de la communauté. Il est vrai que la métamorphose de Capanée, le soudard impie des Sept contre Thèbes justement foudroyé par Zeus, en un membre estimé de la société, a fait douter du sérieux d'Euripide dans l'ensemble du morceau, qu'on a considéré comme un pastiche de l'éloquence officielle ${ }^{63}$. Mais Capanée présente ici un cas particulier, car l'époux d'Évadné doit justifier par ses mérites la passion de la jeune femme. Ajoutons qu'Euripide aime assez à surprendre son public en « réhabilitant » des héros ou des héroïnes maltraités par ses prédécesseurs, comme Hélène ou la Clytemnestre d'Ipbigénie à Aulis. D'une façon générale, je croirais qu'Euripide, dans ce morceau, a voulu suggérer une manière d'améliorer un genre quelque peu artificiel pour qu'il remplisse plus sûrement sa fonction civique.

On remarquera en tout cas que l'éloquence d'Adraste n'a aucun effet apaisant sur les Mères, qui se livrent aussitôt après à une de leurs explosions de douleur les plus vives et les plus spontanées (918-924) ${ }^{64}$ !

Quant à Evadné, il est clair qu'Euripide a présenté son comportement comme l'opposé de celui d'une veuve de guerre athénienne : elle se présente en vêtements de fête, elle veut rivaliser avec les exploits de son mari (1059-1063), et au lieu de se tenir en larmes auprès du bûcher funéraire, elle se jettera sans hésiter au milieu du brasier pour rejoindre son mari «dans les chambres de Perséphone » (1022). Evadné s'ajoute dans le monde des héros aux figures des grandes amoureuses, Laodamie, la veuve de Protésilas, ou Alceste, mais avec une sorte d'emportement délirant que condamne le Chœur (1072). Il est vrai que la figure de Capanée est elle-même hors des normes, puisqu'il a été frappé par la foudre de Zeus, qui est une sorte de signe d'élection. Cela lui sera reconnu, puisque comme héros bienfaisant, il sera enseveli à part dans l'enceinte même du sanctuaire des deux déesses, sur une terre sanctifiée par le feu que le poète

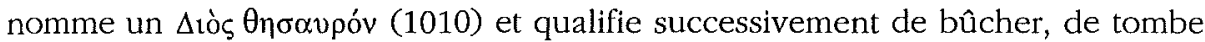
et de chambre funéraire $(980-981 ; 1010 ; 1058)^{65}$.

62 Exemple tragique le plus développé : Hér., 348-450 (le Chour célèbre Héraclès qu'il croit mort); Crespbonte, fr. 67 Austin.

63 Voir J.W. FITTON, in Hermes, 89 (1961), p. 430 sq.; D.J. CONACHER, p. 24-25; SMITH, p. 162-163. Désaccord de COLLARD, op. cit. (n. 59), p. 44.

64 Comme le souligne CONACHER, p. 25. - Je ne suis pas N. LORAUX, L'Invention..., p. 49, selon laquelle le discours d'Adraste aurait pour effet « d'intégrer pour la première fois les Mères dans l'univers civique »; cf. MIRTO, p. 84-87.

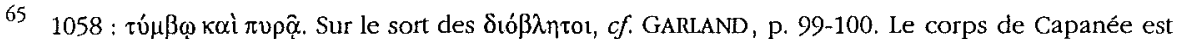
devenu ípós, ainsí que son tombeau (935 et 981). Comme le héros Pélops à Olympie, il peut être inhumé dans l'enceinte sacrée. 
Les thèmes de la lamentation rituelle sont à la fois simples et peu nombreux; certains sont aussi vieux que la poésie homérique ${ }^{66}$. La déploration se développe autour de deux pôles : avant et après, le bonheur d'autrefois et le malheur de demain, avec une sorte d'épicentre où la souffrance présente peut s'épancher grâce au rite. Selon la loi commune du genre, la plainte est avant tout personnelle, mais c'est aussi une manière de faire sentir au mort combien sa présence était importante pour ceux qu'il vient de quitter ${ }^{67}$. D'un morceau à l'autre, les mêmes thèmes sont repris, aussi bien par les Mères, que par d'autres victimes : l'épouse privée de son mari, le père privé de ses enfants, les enfants privés de leur père.

Tout naturellement, c'est vers le passé que se tournent ceux qui pleurent, un passé nimbé de bonheur. Les mères ont accompli leur fonction naturelle en mettant au monde des fils, sous l'égide d'Artémis Lokhia (957-958). De beaux enfants, bien sûr (955-957). C'étaient «les plus nobles des Argiens » (965), «glorieux à Mycènes » (1131). Elles insistent sur les liens uniques qui réunissent mère et enfant, depuis la grossesse et les douleurs de l'enfantement ${ }^{68}$, puis avec les soins de la petite enfance, y compris même les nuits sans sommeil (1137) ${ }^{69}$. Les baisers affectueux $(1138)^{70}$ expriment cette intimité physique. Pour sa part, le père d'Étéoclos et d'Evadné se targue d'avoir eu un « noble fils » (1092-1093), mais il se souvient surtout de l'amour de sa fille, «cette enfant qui couvrait ma joue de baisers et qui prenait ma tête entre ses mains » (1099-1101) ${ }^{71}$. Le jeune fils, de son côté, se souvient du visage de son père, de sa voix qui l'exhortait et sa grand-mère lui rappelle « le doux baiser qu'il posait sur (sa) joue » (1153-1156).

Le contraste entre le bonheur passé et la douleur présente est tel qu'on voudrait que ce passé n'ait pas eu lieu : «le Temps aurait dû me préserver du mariage », dit une mère, «car à quoi bon les enfants? » (786-789). De même, Iphis se repent d'avoir passionnément désiré des enfants, pour s'en trouver désormais privé $(1087-1093)^{72}$.

Quant à l'avenir, pour les parents comme pour les enfants, c'est le domaine de la privation, de la solitude, du désespoir ${ }^{73}$. La perte des enfants est un mal pire que le veuvage (790-793). La mère, dépossédée ne peut envisager qu'une vieillesse stérile, solitaire, méprisée par rapport aux mères fécondes (958-959), peut-être même en proie à la gêne matérielle, faute de fils pour « nourrir sa vieil-

REINER, p. 14-23. Lamentation d'Andromaque, Il., XXII, 477-514; XXIV, 715-745.

67 REINER, p. 22-23.

68 919-922, 1135-1136; cf. Méd., 1029-1031, 1262; Tr., 760; Hyps., fr. 60, 1, 1-12.

69 Ion, 1377; Tr., 1187; pour les nuits sans sommeil, ESCH., Cho., 751; EUR., Tr., 1188.

70 Méd., 1074; Hếc., 409-410; Tr., 757, 761.

71 Ajouter I.A., $1222 \mathrm{sq.}$

72 Cf. l'"̌p $\omega \varsigma \pi \alpha i ́ \delta \omega v$ de Créuse et Xouthos, Ion, 67; And., 918, 1205.

73 Sur le motif de l'épquía, 1095-1096, 1132-1133; IT, 707; Pbén., 275. 
lesse $^{74}$ ». Cette déchéance est traduite par une série d'adjectifs de sens privatif ${ }^{75}$. Désormais privée de point fixe, sa vie est «comme la nuée errante, ballottée par les vents d'orage », c'est « une vie qui n'est pas une vie» $(960-962)^{76}$.

Plus sombre encore est le désespoir d'Iphis, frustré de toute sa descendance. Il renonce à vivre dans un foyer désert : au fond de sa demeure, dans les ténèbres, il se laissera mourir de faim. Une telle solution ultime, qui n'est pas sans exemple dans la tragédie ${ }^{77}$, est le terme logique à ses yeux d'une existence qui n'a plus d'utilité sur cette terre (1112). Les enfants des Sept se sentent aussi abandonnés dans un foyer désert (1132-1133). Mais, à la différence des vieillards, ils sont au début d'une vie au cours imprévisible. De fait, au moment même où ils ensevelissaient leur père s'éveille en eux l'espoir de se venger un jour sur leurs assassins, un rêve qui apporte un peu de lumière dans les ténèbres de ce sinistre $\operatorname{commos}^{78}$. La promesse d'Athéna dans l'exodos viendra fortifier cet espoir (1144$1147 ; 1150-1152$ ).

Entre le passé et l'avenir, le moment des funérailles est celui du paroxysme de la douleur, exacerbée par la présence même des restes des morts. Le caractère insoutenable de cette souffrance est traduite par l'exclamation des Mères dans le dernier commos : "Ma misère ne peut encore s'endormir : assez pour moi de pleurs, assez de sanglots, assez d'infortunes !» (1147-1149). Mères et fils sont de la même façon douloureusement conscients du contraste entre la légèreté de la cendre qui remplit l'urne funéraire et le poids du chagrin qui pèse sur eux ${ }^{79}$. C'est alors qu'ils ressentent la réalité de cette séparation de l'âme et du corps déjà évoquée par Thésée. D’où ce double gémissement : « LES ENFANTS. Ils sont partis, ils ne sont plus, hélàs ! mon père, ils sont partis ! - LEs MÈres. L'éther désormais les possède, consumés dans la cendre du bûcher. Ils se sont envolés pour gagner l'Hadès ${ }^{80}$ ». Il est naturel que les Mères expriment le vœu de mourir pour rejoindre les êtres chers dans l'Hadès ${ }^{81}$. C'est là un motif presque obligé du

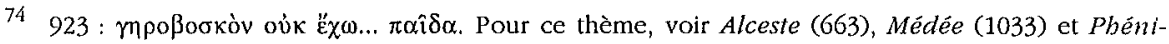
ciennes (Phén., 1436).

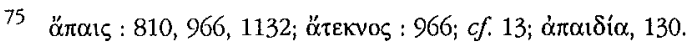

76 Cette incertitude de vie est explicitée dans l'antistrophe (968-969). Pour l'état entre vie et mort, Alc., 141; Hëc., 431; El., 201-202; Tr., 1223.

77 Ce sont dans la tragédie les deux signes du désespoir le plus profond, mais ils ne vont jamais jusqu'à la mort (Méd., 24; Hipp., 196, 275; Or., 41, 189).

78 Le désespoir conduit naturellement aux pensées de vengeance. Mais la promesse de nouveaux carnages est-elle vraiment un espoir salutaire? Le poète était en tout cas lié par le mythe.

79 Souvenir évident de l'Agamemnon, $437 \mathrm{sq}$., avec une antithèse de nature différente.

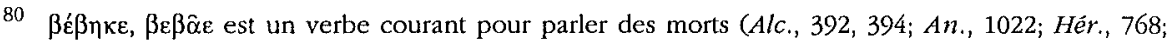
Or., 971); pour les âmes des morts qui s'envolent vers l'Hadès, déjà $O d$., XI, 222; elles sont figurées sur les lécythes à fond blanc par de petites créatures ailées voletant au-dessus de la tombe ( $c f$. G. SIEBERT, Eidôla, in Méthodologie iconograpbique, Strasbourg, 1981, p. 63-73).

81 769-797. Voir E. VALGiglio, Il tema della morte in Euripide, Turin, 1966, p. 51-52. 
thrène. Seule Evadné en mène la réalisation jusqu'à son terme, comme l'unique destin concevable pour elle ${ }^{82}$.

Adraste a partagé sans retenue les manifestations du chagrin des Mères, ce que Thésée a paru désapprouver. En même temps, il a tenu plusieurs fois à souligner que leur deuil est aussi celui de la cité tout entière. Il appelle même la mort sur lui, mais l'expression de son désespoir est plus rhétorique que convaincante (821-831). Cependant, ses exclamations tirent un moment les Mères de leur propre chagrin, pour les amener à compatir au sien: «Amères furent les noces (de tes filles), tu l'as vu; amer, l'oracle de Phoïbos. L'Erinye chargée de pleurs a déserté la maison d'CEdipe pour venir à toi ! » $(832-836)^{83}$.

L'exodos confirme l'achèvement des rites en y ajoutant, comme il est fréquent chez Euripide, plusieurs aitia, liés en partie à Éleusis. En effet, alors que

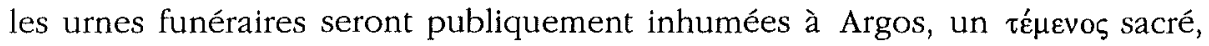
une enceinte interdite, conservera sur le territoire éleusinien le souvenir de la crémation des chefs, «là où les corps furent purifiés par le feu » $(1211)^{84}$. Le lieu, qui devait être connu des Athéniens, est précisé, au carrefour de la route de Thèbes et de celle de Corinthe ${ }^{85}$. Euripide raccorde ainsi, avec quelque laxisme, son récit à une tradition locale qui conservait le souvenir des sept tombeaux des chefs argiens. Ici, Athéna parle seulement des sept bûchers funéraires, alors que Thésée avait fait édifier un bûcher collectif pour quatre des cinq corps, Capanée ayant sa tombe dans le sanctuaire. Mais l'attention du public dans l'exodos doit être ramenée à la légende thébaine, avec la mort des sept chefs vengée une génération plus tard par les sept épigones. Quant aux Mères, apaisées par l'aboutissement du rite et les paroles bienveillantes d'Athéna, elles sont prêtes à témoigner leur reconnaissance à Thésée et aux Athéniens (1232-1234).

Ce qui met la lamentation funèbre des Suppliantes au-dessus des rites de la vie quotidienne, c'est sa profonde élaboration artistique. Elle s'inscrit dans une tradition tragique où Eschyle tient la première place. Malgré l'interdiction de Solon, dans les funérailles du $v^{e}$ siècle on exécutait des thrènes préparés à l'avance, et il existait une poésie funéraire. Mais ces chants se retrouvent stylisés et magnifiés, entre autres dans les trois grands commoi du théâtre d'Eschyle, ceux des Perses, des Sept et des Choéphores, dont l'influence sur les cantica des

82 Voir N. LORAUX, Façons tragiques de ther une femme, Paris, 1985, p. 54, 59, qui met l'accent sur le motif du « mourir-avec » : noter la répétition du préfixe ovv-dans le passage (1007, 1014, 1020, 1029, et aussi 1063 et 1071).

83 Sur l'Érinye d'Edipe, ESCH., Sept, 987-988.

84 Pour le pouvoir purificateur du feu sur les corps, Hér., 937; Or., 40 et sch.

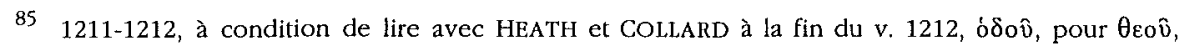
leçon qui a suscité de très nombreuses exégèses, en général peu satisfaisantes; voir éd. COLLARD ad loc, et pour les realia F. JACOBY, FGrH IIIb, Suppl. 2, p. 351, n. 23. 
Suppliantes a été souvent notée ${ }^{86}$. On a retenu en particulier l'usage des introductions anapestiques, l'allure fortement cadencée du chant, avec de nombreuses anaphores, les constants échos entre strophe et antistrophe - de sonorités, de mots et d'idées - la véhémence de l'expression. Celle-ci se traduit par les exclamations douloureuses, les cris d'imploration ou d'exhortation, l'interpellation directe des morts, etc. Le mouvement est accentué par la séparation du Chœur en deux groupes (second stasimon), et, fait plus exceptionnel, par le dialogue du second commos entre deux chœurs. La beauté de l'expression et du chant affine ainsi le pathétique. Si on compare ces cantica à ceux d'Eschyle, on note un adoucissement du ton. La brutalité des manifestations physiques du deuil s'atténue, ainsi que la violence des cris. La douleur maternelle semble s'exprimer ici sans entraves, mais elle est en fait bridée par le développement des conventions sociales dans l'Athènes du dernier quart du $v^{e}$ siècle.

Ainsi s'achevait dans l'apaisement une aventure tragique. Il revenait à la plus civilisée des cités grecques de rétablir l'ordre des dieux et des hommes dans un monde hérö̈que perturbé par les folles ambitions d'Argos et l'bybris de Thèbes. Aussi les Argiens tombés devant la Cadmée obtenaient-ils, comme les Athéniens morts devant Délion, les honneurs funèbres qui leur étaient dus, et les mères argiennes, comme les mères athéniennes, trouvaient dans leur participation aux rites le réconfort que donne l'accord avec les coutumes ancestrales ${ }^{87}$. Mais, dans les Suppliantes, le mode des funérailles des Sept, calqué sur des modèles ambigus, de caractère à-demi public à-demi privé, traduit le flottement de la cité entre un type de cérémonie hérité de la tradition et dominé par le déchaînement des manifestations de douleur féminines, et un autre type de célébration, ouverte à tous, mais ordonnée par les responsables masculins de l'État, où la souffrance était maîtrisée et où dominait la leçon morale offerte par ceux qui avaient donné leur vie pour la patrie. Euripide, le poète de la douleur et de la faiblesse humaine, était porté par sa sensibilité à comprendre et à traduire le chagrin provoqué par la mort d'un combattant chez les femmes de sa famille. Mais aussi, il a voulu montrer qu'un bon usage de cette institution publique qu'était l'oraison funèbre des morts au combat était propre à renforcer la solida rité de tous les Athéniens dans les temps d'épreuve.

François JoUAN

46, rue Saint-Pierre

F - 14000 CAEN

86 Nous nous bornerons ici à un unique exemple, celui de l'adjectif $\alpha i \mu \alpha \tau o \sigma \varphi \alpha \gamma \eta ́ s$ (812), qui ne se trouve que dans les Perses (816), et qui est plus employé pour sa valeur poétique que pour son exactitude dans le contexte de la pièce.

87 Voir les justes remarques de M.S. MIRTO, p. 86-87, sur la «culture des mères » opposée aux valeurs de la cité. 